\title{
STRUKTURELLE, SEMANTISCHE, FUNKTIONALE UND STILISTISCHE PARAMETER DER VERBALEN INNOVATIONEN DER DEUTSCHEN SPRACHE
}

\author{
Anna Zavgorodnia \\ Bewerberin, Nationale Linguistische Universität Kiew, die Ukraine \\ e-mail: annazando@gmail.com,orcid.org/0000-0001-5458-9656
}

\section{Zusammenfassung}

Der vorliegende Artikel befasst sich mit der Frage der strukturellen, semantischen, funktionalen und stilistischen Parameter der verbalen Innovationen aus der deutschsprachigen Publizistik. Der Artikel betrachtet die neuen Verben vom Standpunkt der Neuigkeit ihrer Form und Inhalt aus. Die Untersuchung erörtert alle wortbildenden Mittel, die an der Erscheinung von den neuen Verben beteiligt sind. Auf der Grundlage von der durchgeführten Analyse werden die produktivsten wortbildenden Einheiten erwähnt. Von großer Bedeutung ist die Frage der lexikalisierten Verben, die in die Wörterbücher übergegangen sind. Das Funktionieren der neuen Verben in dem publizistischen Stil beschränkt sich auf die Untersuchung der wertenden Bedeutungen der einzelnen Bestandteilen der Wörter und selbst der verbalen Innovationen im Großen und Ganzen, auf das metaphorische Gebrauch der Verben und auf die Fehler im Gebrauch der grammatischen Kategorien des Verbs wie Person, Numerus, Tempus, Genus und Modus. Hier sei noch betont werden, dass die bewertenden Konnotationen der Verben rein subjektiven Charakter haben und stark von dem Kontext abhängen.

Schlüsselwörter: Verb, Innovation, Wortbildung, Publizistik, Bedeutung, Bewertung.

DOI: https://doi.org/10.23856/4014

\section{Einleitung}

Jede Sprache ist wie ein Lebewesen, das lebt und sich ständig entwickelt. Die Sprache widerspiegelt alles, was in der Welt passiert: alle politischen, sozialen, wirtschaftlichen und wissenschaftlichen Änderungen finden ihren Spur in der Sprache. Neuerscheinungen, neue Gegenstände und einfach neue Namen statt der alten werden verbal ausgedrückt.

Neue Lexik oder neue Wörter erscheinen jeden oder fast jeden Tag. Neue Wörter werden in der Sprachwissenschaft entweder Neologismen oder Innovationen genannt. Die meisten neuen Wörter sind die Nomen. Viel seltener tauchen Verben und Adjektive auf. Unsere Interesse betreffen nur die neuen Verben, weil der Nomen und das Verb die Hauptpositionen im Satz besitzen und weil die Nomen schon von vielen Wissenschaftlern untersucht worden sind.

Die meisten neuen Wörter erscheinen in der Umgangssprache oder im Internet, in den Zeitungen und Zeitschriften. Aus diesem Grund werden die neuen Verben aus der deutschsprachigen Publizistik der vorliegenden Arbeit zugrunde gelegt.

Die Fragen der neuen Verben in der deutschen Sprache haben folgende Sprachwissenschaftler wie L. I. Zima, L. B. Wolowyk, S. W. Druschbjak, N. P. Smoljar, T. I. Skorobogataja schon beleuchtet.

Das Objekt der Arbeit stellen die Bestandteile der Verben (Morphemen), ihre lexikalischen (Semen) und stilistischen Bedeutungen dar.

Als das Gegenstand der vorliegenden Untersuchung dienen deutschsprachige Zeitschriften und Zeitungenund ihre Internetseiten. 
Die Aktualität unserer Forschung besteht in der Untersuchung von den neuen Verben, die neulich erschienen sind, und die die Tendenzen und die Entwicklungsgesetzmäßigkeiten der Deutschen Sprache, die Erweiterungswegen des Wortschatzes und die Expressivität der Sprache feststellen können.

Das Ziel des Artikels liegt in der Forschung der semantischen, strukturellen, funktionalen und stilistischen Merkmale von den Verben-Innovationen. Es werden folgende Aufgaben in den Vordergrund gestellt: 1) die Besonderheiten der Innovationen beschreiben; 2) die wortbildende Mittel betrachten und die produktivsten Modelle nennen; 3) die stilistischen Besonderheiten der Verben in der deutschsprachigen Publizistik beleuchten.

Der Artikel lehnt sich methodisch an wissenschaftliche (Analyse, Systematisierung, Klassifikation), linguistische (beschreibende, struktur-semantische) und mathematische Methoden an.

In dieser Arbeit werden 827 neuen Verben aus den deutschsprachigen Zeitungen und Zeitschriften präsentiert. Sie werden den deutschsprachigen Zeitungen und Zeitschriften wie «Der Spiegel», «Süddeutsche Zeitung», «Bild», «die Zeit», «RP», «Creditreform», «Netzwelt», «Der Tagesspiegel», «Lerntippsammlung», «Ad-hoc-News», «Die neue Osnabrücker Zeitung»; «Der Standard», «Heute», «Kleine Zeitung»; «20 Minuten» entnommen. Von Bedeutung sind die strukturellen, semantischen und funktionalen Parameter.

\section{Die Natur der Innovation}

Unter dem Begriff Neologism versteht man alle neuen Wörter, die im Wörterbuch nicht eingetragen sind, werden aber von den Menschen verstanden.

Jedes neue Wort macht vier Schritte, um in ein Wörterbuch zu geraten, und zwar: Erscheinung, Usualisierung, Akzeptualisierung und Lexikalisierung. Die Erscheinung des Wortes ist durch die Benennung von neuen Denotaten (Erscheinungen und Gegenständen) gekennzeichnet. Das ist die erste Benennung des Wortes. Usualisierung bedeutet die Wahrnehmung der Innovation von den Sprachlern, wenn die meisten Sprachbenutzer die Bedeutung des Wortes verstehen. Akzeptualisierung ist der nächste Schritt, wenn das neue Wort den grammatischen Regeln der neuen Sprache anpasst. Und der letzte Schritt im Leben der Innovation ist die Lexikalisierung oder die Integration, die das Eintragen des Wortes in das Wörterbuch bedeutet. Nach diesem Schritt endet sich „das Leben“ der Innovation. Danach übergeht das Wort in den gemeinsamen Wortschatz der Sprache (Lysezka, 2003: 36).

Es gibt verschiedene Klassifikationen der Neologismen. Die umfangreichste Klassifikation wurde von N. W. Murawljowa und E. W. Rosen vorgestellt. Sie betrachten alle neuen Wörter vom Standpunkt der Semantik (Inhalt) und der Form aus:

- Neuwörter;

- Neuprägungen oder Neuformative;

- Transnominationen;

- Neubedeutungen.

Neuwörter sind neu sowohl visuell (Form) als auch inhaltlich. Sie bezeichnen ganz neue Erscheinungen oder Gegenstände, die früher nicht existierten. Neuprägungen oder Neuformative bezeichnen neue Wörter, die auch neue Denotate nennen, aber aus schon bekannten Wortteilen bestehen (Präfixe, Suffixe, Halbpräfixe, Wortstämme). Sie haben neue Semantik und relativ neue Form. Transnominationen sind neue Wörter, die schon bekannte Erscheinungen und Gegenstände mit den neuen Wörtern benennen. Sie haben neue Form und nicht neue Bedeutung. Neubedeutungen sind Wörter, die entweder ihre Bedeutung geändert haben, oder in einen anderen funktionalen Stil übergegangen sind. Sie haben neue Bedeutung und alte Form. Folgende Tabelle bildet diese Arten der Neologismen mit den Beispielen ab (Murawljowa, 1978: 7-8; Rosen 1971: 42-43). 
Die Neuigkeit der Innovationen nach der Form und dem Inhalt (Semantik)

\begin{tabular}{|l|c|c|l|}
\hline & Form & Inhalt & \multicolumn{1}{|c|}{ Beispiele } \\
\hline Neuwörter & + & + & croonen, kickboxen, trixen, stalken \\
\hline Neuprägungen oder Neuformative & + & + & zutexten, spuckpusten, abchillen \\
\hline Transnominationen & + & - & $\begin{array}{l}\text { SMSen - SMS schreiben, heimwalken - } \\
\text { nach Hause gehen }\end{array}$ \\
\hline Neubedeutungen & - & + & surfen, umwälzen \\
\hline
\end{tabular}

\section{Srtuktur und Semantik der verbalen Neologismen}

Die formale und strukturelle Seiten des Wortes bilden die Basis der Untersuchung von den strukturellen und semantischen Parameter der Neologismen. Die Struktur des Wortes wird durch die Wortbildung erörtert. Die Wortbildung ist eine Wissenschaft, die eine Zwischenposition unter den Sprachniveaus (Morphologie, Lexikologie und Syntax) benimmt. Neue Wörter erscheinen in der Sprache mit Hilfe der folgenden wortbildenden Mittel wie Affigierung (Präfigierung, Halbpräfigierung, Suffigierung), Zusammensetzung, Verbalisierung und gemischter Typen (Zima, 1981: 42, Wolowyk, 2012: 33-34).

Das produktivste Mittel ist die Affigierung (es gibt $37 \%$ der neuen Verben, die nach diesem Modell gebildet wurden) und zwar die Halbpräfigierung (43\% der Verben, die anhand der Affigierung gebildet wurden). Die Frage der Halbpräfigierung ist unter vielen Wissenschaftlern ein Streitpunkt. Es gibt unterschiedliche Standpunkte, was man zu den Halbpräfixen zählen kann. Unserer Ansicht nach gehören zu den Halbpräfixen alle nicht selbstständigen Wortarten wie Präpositionen. Die Präpositionen können von den anderen Wissenschaftlern entweder zu den ersten Teilen der Zusammensetzungen oder zu den Präfixen eingeordnet werden (Schewelewa, 2004: 62-63, Druschbjak, 2010: 95, Skorobogataja, 2011: 111).

Jedes Halbpräfix hat seine eigenen Bedeutungen, die die Bedeutung des neuen Wortes entweder bereichern, oder ändern. Zu den produktiven Halbpräfixen gehören mit-, nach-aus-, zu-, bei-, an-, ab-, auf, unter-, gegen-, über-, vor-, dutch-, auf-, entlang-, um-, z.B: mitsimsen, aufriestern, durchsurfen, gegenregulieren, abkichern, umklicken, nachcasten, ausflexen, zumailen, beibrüllen, anspammen, vorscrollen, überkalendern,runtermerkeln. Außerdem wurden auch die nicht neuen Verben (aus dem Wörterbuch) analysiert und es hat sich noch andere Bedeutungen / Schattieerungen der Halbpräfixen ergeben, die bei den Innovationen nicht mitgemacht haben. Ansonsten gibt es auch eine Gruppe der Präpositionen wie gegenüber-, entgegen-, wider-, entlang-, hinter-, die unter den neuen Verben nicht gefunden wurden. Es kann vermutet werden, dass sie gar unproduktiv bei der modernen verbalen Wortbildung sind.

Die Präfigierung ist in der deutschen verbalen Wortbildung nur bei den verbalen Präfixen be-, er-, ver-, zer-, ent-, (ge-, miss- und emp- sind jetzt nicht produktiv) und bei den entlehnten Präfixen wie de-, re-, dis-sub- präsentiert, z.B: bequoten, verdoodeln, zerdiskutieren, ershoppen, entdenglischen, deabonnieren, republizieren, disruptieren, sublizensieren. Die produktivsten Präfixen sind ver-, ent-, re-, be-, de-, er-, zer-, dis-. So wie die Halbpräfixe können Präfixe die Bedeutung des neuen Wortes je nach der Situation (Kontext) entweder verstärken oder verändern. Die produktivste Komponente unter den allen ersten Komponenten ist Präfix VER-.

Es lässt sich erwähnen, dass Präfigierung und Suffigierung nach der Produktivität auf demselben Platz sind. Es sind je 86 neue Verben gefunden, die mit dem Präfix und dem Suffix gebildet wurden. Das produktivste Suffix ist -ier(en) und seine Formen -isier(en) und -(i) fizier(en) (pogromieren, egofizieren). Nicht so produktiv sind -er(n) und -el(n) (jaggern, monumenteln). Alle anderen deutschen Suffixe sind gar nicht produktiv. Es sind keine verbalen Neologismen mit den anderen Suffixen gefunden. 
Die Zusammensetzung hält man für das produktivste Wortbildungsmittel in der deutschen Sprache (Gorbatsch, 2014: 116), aber nicht bei den Verben. Hier besitzt sie nur den 2. Platz. Es sind 26\% der neuen Verben, die mit Hilfe der Zusammensetzung gebildet wurden. Die meisten neuen Verben bestehen aus zwei Komponenten, die erste von denen entweder Adjektiv/Adverb oder Nomen (seltener) auftaucht. Die zweite Komponente soll unbedingt ein Verb sein. Es wurde bemerkt, dass einige Komponente in der Bildung von vielen Verben beteiligt haben. In der Linguistik heißen sie häufige Komponente. In unseren Untersuchng gehören zu den häufigen Komponenten quer-, zurück-, hoch-, weg-, zwang-, -googeln, -klicken und andere, z.B. hochgoogeln, zurückalbern, zwangsenthaaren.

Noch $22 \%$ der neuen Verben wurden mit Hilfe der Verbalisierung hervorgebracht. Die meisten von denen wurden entlehnt. Die andere Benennung von der Verbalisierung ist Konversion. Die Aufgabe der Konversion liegt in der Verwandlung der nominalen Wortarten in Verben oder in der Anpassung der entlehnten Verben an die grammatischen Regeln des Deutsch, z.B. hochdeutschen, developen, powerpointen.

Die wenigsten Verben wurden anhand der gemischten Typen geformt. Zu den Gemischten Typen gehören folgende: Präfigierung + Suffigierung, Halbpräfigierun + Suffigierung, Präfigierung + Konversion, Halbpräfigierung + Konversion, Präfigierung + Zusammensetzung, Zusammensetzung + Konversion, Zusammensetzung+Suffigierung,Präfigierung+Zusammensetzung+Verbalisierung. Darüber hinaus gibt es noch ein Typ, der aber unter den verbalen Innovationen nicht präsentiert ist - Halbpräfigierung + Zusammensetzung. Die produktivsten Modelle sind Präfigierung + Konversion, z.B. verskillen, Zusammensetzung + Konversion, z.B. zurückleaken, und Präfigierung + Suffigierung, z.B. entpseudonymisieren.

Die Zeitraum, wann die Innovationen gesammelt wurden, beträgt 18 Jahre. Während dieser Periode wurden viele Innovationen lexikalisiert, d.h. sie haben aufgehört, Innovationen zu sein und sind in das Wörterbuch eingetragen. Es soll betonnt werden, dass die meisten lexikalisierten Verben durch Verbalisierung (Konversation) $-31,9 \%$ und Affigierung - 11,5\% erschienen sind. Der gemeinsame Anteil der lexikalisierten Innovationen beträgt 13\%. Unten gibt es Tabelle, die das beleuchtet.

Anzahl der lexikalisierten Innovationen

\begin{tabular}{|l|c|c|c|}
\hline \multicolumn{1}{|c|}{ Wortbildungstyp } & $\begin{array}{c}\text { Anzahl der } \\
\text { Einheiten }\end{array}$ & $\begin{array}{c}\text { Anzahl der } \\
\text { lexikalisierten } \\
\text { Einheiten }\end{array}$ & $\begin{array}{c}\text { \% der } \\
\text { lexikalisierten } \\
\text { Einheiten }\end{array}$ \\
\hline Affigierung & $\mathbf{3 0 2}$ & $\mathbf{3 5}$ & $\mathbf{1 1 , 5 \%}$ \\
\hline -Präfigierung & 86 & 12 & $14 \%$ \\
\hline -Halbpräfigierung & 130 & 7 & $5,4 \%$ \\
\hline -Suffigierung & 86 & 16 & $18,6 \%$ \\
\hline Zusammensetzung & $\mathbf{2 1 5}$ & $\mathbf{1 0}$ & $\mathbf{4 , 7 \%}$ \\
\hline Verbalisierung & $\mathbf{1 8 2}$ & $\mathbf{5 8}$ & $\mathbf{3 1 , 9 \%}$ \\
\hline Gemischte Typen & $\mathbf{1 2 8}$ & $\mathbf{5}$ & $\mathbf{3 , 9 \%}$ \\
\hline - Präfigierung+Suffigierung & 16 & 0 & $0 \%$ \\
\hline - Halbpräfigierung+Suffigierung & 5 & 0 & $0 \%$ \\
\hline - Präfigierung+Verbalisierung & 40 & 2 & $5,2 \%$ \\
\hline - Halbpräfigierung+ Verbalisierung & 22 & 3 & $13,7 \%$ \\
\hline - Präfigierung+Zusammensetzung & 3 & 0 & $0 \%$ \\
\hline - Zusammensetzung+Verbalisierung & 33 & 0 & $0 \%$ \\
\hline - Zusammensetzung+Suffigierung & 8 & 0 & $0 \%$ \\
\hline - Präfigierung+Zusammensetzung+ Verbalisierung & 1 & 0 & $0 \%$ \\
\hline ZUSAMMEN & $\underline{\mathbf{8 2 7}}$ & $\underline{\mathbf{1 0 8}}$ & $\underline{\mathbf{1 3 \%}}$ \\
\hline
\end{tabular}


Bei der Untersuchung der strukturellen Parameter der verbalen Innovationen wurde auch auf die häufige Komponente eingegangen. Die Analyse beschränkt sich auf 3 Gruppen von den häufigen Komponenten, und zwar die am Anfang, in der Mitte oder am Ende des Wortes stehen. Am häufigsten tauchen die letzten Komponente (45\%), am seltensten - in der Mitte (16\%) auf. Unter den ersten Komponenten sind das deutsche Präfix ver- (50 Verben), Halbpräfixe auf- (23), $a b$ - (21) und die ersten Komponente in Zusammensetzungen zurück- (25), weg- (20) am aktivsten. Die letzten häufigen Komponente sind nur in 77 Verben vertreten. Sie lassen sich von 2 (53 Fällen) bis 12 mal (- klicken) in verschieden Innovationen sehen. Die Anzahl der Komponente in der Mitte des Verbes beträgt nur 27 in 90 Verben (-fern-, -frei-, -schnack-, -blog-, -power-, -cyber-).

\section{Stilistische Besonderheiten der verbalen Neologismen}

Für die Forschung der funktionalen und der stilistischen Parameter von den verbalen Innovationen wurden die bewertende Bedeutung der Verben, ihre metaphorische Benutzung und die Brechung in der Benutzung von den grammatischen verbalen Kategorien auseinandergesetz. Die bewertende Bedeutung des Verbes hat subjektiven Charakter und hängt oft vom Autor ab und kann entweder offen oder latent bezeichnet werden. Die wertende Konnotation des Verbs kann positiv, negativ oder neutral sein. Selbst die Bewertung findet sich in dem ganzen Wort oder in seinem Bestandteil, deshalb wird die Bewertung sowohl in den einzelnen Bestandteilen des Verbs als auch in dem ganzen Wort in Betracht gezogen. Dafür werden alle verbalen Neologismen in 27 bewertenden Gruppen aufgeteilt, unter denen 3 nicht aktiv waren. Verschiedene Kombinationen von den wertenden Konnotationen der Wortteilen können sich in verschiedenen Bedeutungen des ganzen Wortes ergeben, z.B. "negativ + negativ = negativ" - krankjammern, "negativ + negativ = positiv" - disruptieren, "negativ + negativ = neutral" - weglärmen .

Die metaphorische Bedeutung kann in zwei Fällen ausgedruckt werden: 1) grammatisch - das ist die Konversion, wenn sich die nominalen Eigenschaften in die verbalen verwandeln; 2) semantisch - wenn das Verb eine neue, oft übertragene Bedeutung bekommt (Smoljar, 2010: 8), z.B. «Bald gibt es keine mündigen Menschen mehr in diesem Land, weil die Politik bis ins kleinste alles regelt und nannysiert hat» (Zeit online, 2018).

Die grammatischen Besonderheiten des Verbs, die semantischen Charakter haben, kommen in der "falschen" Benutzung der grammatischen Kategorien zum Ausdruck. Es sei auch erwähnen, dass das Verb folgende Kategorien besitz, wie: Person, Numerus, Modus, Genus und Tempus. Z.B., im Sazt «Der Nepalese Rabi Lamichhane will einen neuen Guinness-Rekord setzen. Und dafür 60 Stunden durchtalken» (Der Tagesspiegel, 2018) wird es auf den Subjekt im zweiten Teil verzichtet. Oder im Sazt «Parkkarte unten rechts einfuddeln» (Spiegel online, 2018) wird Imperativ durch Infinitiv ersetzt. Die "falsche" Benutzung der grammatischen Kategorien des Verbs dient für Sprachökonomie, Vereinfachung der grammatischen Konstruktionen und Erwerben vom stilistischen Effekt, das dem publizistischen Stil sehr eigen ist.

\section{Fazit}

Die oben angeführten Ergebnisse deuten auf die Haupttendenzen der Entwicklung der Neologie in der deutschen Sprache. Es lässt sich feststellen, dass die neuen Verben viel seltener als die Nomen in der Sprache erscheinen, die Publizistik (Zeitungen, Zeitschriften und Internet) stellen die Sphäre dar, wo die neuen Wörter am wahrscheinlichsten auftauchen können. Als Material für die Innovationen dienen sowohl deutsche als auch entlehnte sprachliche Einheiten wie Morpheme und Wortstämme. Für das produktivste wortbildende Modell der deutschen 
Verben hält man Affigierung, und zwar Halbpräfigierung. Selbst die Frage des Begriffs „Halbpräfigierung“ bleibt bis jetzt nicht völlig geklärt. Die Betrachtung der bewertenden Bedeutungen hat rein subjektiven und kontextabhängigen Charakter, das zur Folge hat, dass ein und dasselbe Verb in verschiedenen Situationen kontroverse bewertende Bedeutungen haben kann. Mit dem stilistischen Zweck werden auch grammatische Fehler begangen, was dem Stil der Presse und Publizistik eigen ist. Anhand der angeführten Arbeit sind die Tendenzen der Erweiterung des deutschen verbalen Wortschatzes für die nähere Zukunft sichtbar.

Die nachfolgenden Forschungen der verbalen Innovationen können auch im Stil der Alltagsrede durchgeführt werden und danach die Ergebnisse von beiden Untersuchungen gegenübergestellt werden.

\section{Literaturverzeichnis}

Druschbjak S. W. (2010) Slowoskladannja u tworenni sutschasnych nimezkych ekonomitschuch terminiw [Die Wortbildung bei der Bildung der deutschen wirtschaftlichen Termini]. Lwiw, Wisnyk nazionalnogo uniwersytetu "Lwiwska politechnika" [uaf Ukrainisch].

Gorbatsch O. W. (2014). Skladni neologismy: strukturno-semantytschnyj ta funkzionalno-pragmatytschnyj aspekty [Zusammengesetzte Neologismen: struktur-semantische und funktional-pragmatische Aspekte]. Zaporischschja [auf Ukrainisch].

Lysezka N. G. (2003). Fraseologitschni inniwaziji w sutschasnij nimezkij mowi: kognitywnyj ta funkzionalnyj aspekty [Fraseologische Innovationen in der modernen deutschen Sprache: kognitive und funktionale Aspekte]. Luzk [auf Ukrainisch].

Murawljowa N. W. (1978) Leksitscheskie innowazii obschtschestwenno-polititscheskogo soderschanija $w$ sowremennom nemezkom jasyke [Lexikalische Innovationen des politischgesellschaftliches Inhaltes in der modernen deutschen Sprache ]. Kalinin [auf Russisch].

Rosen E. W. (1971) Nowoe w leleksike nemezkogo jasyka [Das Neue im Deutsch]. Moskau, Prosweschtschenie [auf Russisch].

Schewelewa L. W. (2004) Leksikologija sowremennogo nemeykogo jasyka [Lexikologie der modernen deutschen Sprache]. Moskau: Wysschaja Schkola [auf Russisch].

Skorobogataja T. I. (2011) Osobennosti glagolnogo slowosloschenija w nemezkom jasyke (na primere glagolow fisitscheskogo wosdejstwija) [Die Besonderheiten der verbalen Komposition im Deutsch (am Beispiel der Verben der körperlichen Einwirkung]. Lingwistika i metodika $w$ wysschej schkole [auf Russisch].

Smoljar N.P. (2010) Dijesliwni komposyty w sutschasnij nimezkij mowi: struktura, semantyka, pragmatyka [Verbale Kompositionen im modernen Deustch: Struktur, Semantik, Pragmatik]. Lwiw [auf Ukrainisch].

Wolowyk L. B. (2012) Slowotwirnyj potenzial prefiksalnych dijesliwnych ekonomitschnych terminiw u sutschasnij nimezkij mowi: strukturno-semantytschnyj ta funkzionalnyj aspekty [Wortbildendes Potential von den pr;fixalen verbalen wirtschaftlichen Termini in der modernen deutschen Sprache: struktur-semantische und funktionale Aspekte]. Poltawa [auf Ukrainisch]. Zima L. I. (1981) Glagolnye innowazii i ich ekstalingwistitscheskaja determinirowannost [Verbale Innovationen und ihre extralinguistische Determiniertheit]. Kiew [auf Russisch]. 